\title{
Educación de adultos en determinantes sociales de la salud: Experiencia con la Seremi de Salud de la Región de Los Ríos
}

\author{
ALVARO LEFIO $^{(1)}$, MARÍA TERESA VALENZUELA ${ }^{(2)}$ y NELLY MARCHETTI ${ }^{(2)}$
}

\section{RESUMEN}

Introducción: La necesidad contextual e incremental de generar espacios educativos en torno a los determinantes sociales de la salud (DSS), motivó a la Escuela de Salud Pública de la Universidad de Chile (Espuch) en conjunto con la Secretaría Regional Ministerial de Salud de la Región de los Ríos, a la construcción de un espacio educativo basado en metodologías de educación de adultos y la aplicación de la guía de evaluación de la equidad (GEE). El objetivo de este informe es describir los hallazgos, logros y aprendizajes del proceso. Materiales y Método: Esta sistematización está basada en la planificación del marco de educación de adultos adoptado, el uso del cine en la educación y la utilización de métodos cualitativos para la exploración de percepciones y aprendizajes. Resultados: Se sistematizan las expectativas de los participantes, así como importantes avances en la identificación de determinantes, características del empleo, posibles mecanismos causales involucrados en las inequidades de salud, además de la visualización de grupos vulnerables y barreras implicadas, con mayores dificultades para la reorientación de programas y prácticas laborales cotidianas. Conclusiones y Discusión: La experiencia educativa se mostró como una estrategia facilitadora de aprendizajes para la comprensión de la complejidad de las problemáticas de salud, siendo valorada desde varias perspectivas por los participantes. Se muestra también como un espacio valioso para la comprensión de las dificultades en la implementación de un enfoque local de equidad de los programas de salud y de las prácticas laborales cotidianas del personal sanitario.

Palabras clave: educación en salud, promoción de la salud, factores socioeconómicos, determinantes sociales de la salud, desigualdad, educación de adultos.

\section{ABSTRACT \\ ADULT EDUCATION ON THE SOCIAL DETERMINANTS OF HEALTH: THE EXPERIENCE OF THE REGIONAL MINISTERIAL SECRETARY OF HEALTH, LOS RIOS REGION}

Introduction: The increasing, contextual need for generating spaces for education on the social determinants of health (SDH) motivated the School of Public Health at the University of Chile (SPH-UC) and the Regional Ministerial Secretary of Health of the Los Rios Region, to create an educational methodology based on adult education and applying the Guide for Evaluating Equity (GEE). The goal of this report is to describe the findings, achievements, and lessons of this process. Materials and Methods: This systematization is based on the adoption of a planning

\footnotetext{
(1) Médico Becario Salud Pública. Escuela de Salud Pública. Facultad de Medicina. Universidad de Chile. José Abelardo Núñez 1381. Los Andes. Región de Valparaíso. Chile. lefio.celedon@gmail.com

(2) Escuela de Salud Pública. Facultad de Medicina. Universidad de Chile.
} 
framework for adult education, the use of cinema en education, and the use of qualitative methods to explore perceptions and lessons learned. Results: Results include the systematization of participants' expectations, as well as important advances in identifying determinants, job characteristics, and possible causal mechanisms of health inequity, visualization of the vulnerable groups and barriers involved, in addition to greater difficulties in reorienting programs and everyday work practices. Conclusions and Discussion: Participants'experience showed that the strategy facilitated learning comprehension of complex health problems. It was also shown to be a valued space for understanding the difficulties in implementing a local approach to health equity programs and everyday work practices of health workers.

Key words: health education, health promotion, socioeconomic factors, social determinants of health, inequality, health education

\section{INTRODUCCION}

El enfoque de determinantes sociales de la salud (DSS), ha logrado posicionarse como marco de análisis de los problemas de salud, particularmente desde la formación y posterior informe de la comisión de determinantes sociales de la salud (CDSS) de la OMS ${ }^{1}$ (2007). Dicho enfoque resulta de gran relevancia cuando se habita en uno de los países mas desiguales del planeta como Chile ${ }^{2}$. Diversas iniciativas gubernamentales han recogido este enfoque en el diseño de políticas y programas del ámbito público. La Subsecretaría de Salud Pública del Ministerio de Salud, durante el anterior gobierno, llevó adelante la reformulación de programas nacionales, que -entre otros objetivos- incluía la identificación de grupos vulnerables para la redefinición de prioridades $^{3}$. Desde allí se generaron varias iniciativas de capacitación y formación en DSS, en las cuales diversos académicos de la Escuela de Salud Pública de la Universidad de Chile (Espuch) han participado. Estudiar una de esas experiencias, en la cual la Espuch se involucra en un proceso educativo con la Seremi de Salud de la Región de Los Ríos, es el propósito de este artículo.

Modificar un enfoque no sólo pasa por rediseñar políticas, programas y prioridades, sino que resulta necesario modificar marcos conceptuales y conductuales de las personas que deben implementarlas.

Las vulnerabilidades no se reparten de for- ma homogénea a lo largo de nuestro territorio, por lo cual se requiere que los trabajadores de salud sean capaces de interpretar en forma crítica la compleja realidad en la que están insertas las problemáticas de salud y vincularla con los objetivos esenciales de la política construi$\mathrm{da}^{4}$. En este sentido es que decidimos utilizar la educación de adultos, como metodología facilitadora del aprendizaje y desarrollo de competencias en los trabajadores sanitarios.

La educación de adultos, por su parte, es un concepto con múltiples significados dependiendo del lugar, territorio y grupo humano que la utilice ${ }^{5,6}$. En este artículo se refiere a aquella educación que reconoce el aprendizaje vinculado a la interacción, las emociones y la construcción conjunta del mismo, por lo cual las metodologías a utilizar han de ser activas, contemplarán espacios de diálogo colectivo y de reflexión sobre lo aprendido ${ }^{7}$, además de la solución conjunta de los problemas. Reconoceremos que los adultos, tanto a nivel individual como colectivo, son sujetos con conocimientos, habilidades, valores y actitudes, frutos de su experiencia y base de cualquier actividad educativa $^{8}$.

El objetivo de este informe es describir los hallazgos, logros y aprendizajes del proceso educativo llevado adelante en la Seremi, concebido desde el marco de la educación de adultos, que buscaba fortalecer capacidades comprensivas y prácticas de los participantes, para la reorientación de programas locales de salud en la perspectiva de equidad. 


\section{MATERIAL Y METODO}

\section{1- Breve descripción del proceso educa- tivo}

El proceso educativo fue concebido en cinco etapas: la primera de Planificación, una etapa de Apertura, en que se produjo el encuentro entre el equipo de "expertos" y los participantes, se presentaron los objetivos construidos hasta ese momento y se recabaron las expectativas del los participantes. La tercera etapa la hemos denominado Introducción vincular, y se refiere al primer acercamiento que tuvieron los participantes a los contenidos y en el cual se buscó la vinculación afectiva con ellos, en esta etapa se utilizaron elementos inductivos y de input de las estrategias propuestas por Jane Vella ${ }^{9}$. La cuarta etapa de Aplicación donde se estimuló la integración de los conceptos trabajados en un programa de salud elegido por ellos. La última se refiere a la Evaluación y Sistematización de la experiencia, en la cual exploramos la percepción de los participantes respecto de lo aprendido cuantitativa y cualitativamente, así como se reconstruyó lo realizado en este documento (Figura1).

\section{2-Análisis de necesidades de aprendizaje}

Al momento de iniciarse la planificación del proyecto educativo se contó con las necesidades educativas de la organización destinataria, quienes solicitaron un curso de determinantes sociales de la salud para trabajadores del Departamento de Salud Pública (DSP) y del Departamento de Acción Sanitaria (DAS), que incluía profesionales y no profesionales. Ambos departamentos estarían distribuidos por separado y se solicitó trabajar en base al tema del programa de salud ocupacional. El interés del equipo académico fue poder construir un espacio educativo que permitiese la mejor comprensión de los contenidos y el mayor aporte práctico a la organización. Para ello se eligió poner en práctica la guía de de evaluación de la equidad en programas de salud ${ }^{10}$ (GEE) elaborada por la subsecretaría de salud publica. No se contó ex ante con los intereses de los participantes directos, por lo cual esto fue explorado durante la fase de apertura.

\section{3-Participantes, escenario y tiempo}

A.- Equipo Académico: Estuvo constituido por 3 integrantes: 2 académicas de la Espuch y un estudiante de la especialidad de salud pública, todo lo cual sumó competencias en los ámbitos de: salud pública, educación superior, salud ocupacional, determinantes sociales de la salud, comunicación y metodologías participativas de educación en salud.

B.- Participantes: Al momento de dar inicio al curso se contó con una mayor diversidad de participantes, respecto de su lugar de trabajo, que lo que se pensó inicialmente. Participaron de la experiencia 66 personas pertenecientes a diversos departamentos de la Seremi de Salud. De ellos 68\% era profesional universitario y un $32 \%$ correspondió a personas catalogadas de técnicos o administrativos. El grupo fue dividido en dos, para dos días de capacitación cada uno.

C-Tiempo y Escenario: El curso se desarrolló en 4 días, dos para cada grupo en horario de 9:00 a 17:00 en Valdivia durante noviembre, se utilizó una sala que permitía el trabajo de hasta 5 grupos simultáneamente. 


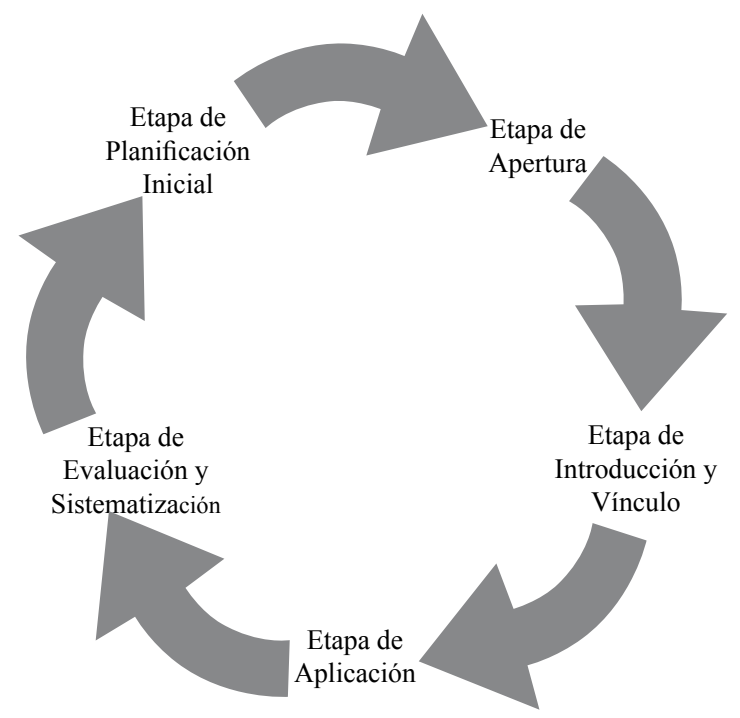

Figura 1. Etapas de la experiencia educativa. Fuente: elaboración propia

\section{4.- Objetivos de aprendizaje}

Los objetivos de aprendizaje definidos previamente al módulo presencial fueron los siguientes: Al final del curso los participantes habrán:

1.- Reflexionado en torno a los DSS y su aplicación práctica en el trabajo cotidiano.

2.- Adoptado nuevas conceptos y categorías de análisis, relacionados con los DSS.

3.- Desarrollado la capacidad de analizar críticamente un programa de salud desde la perspectiva de DSS.

4.- Desarrollado habilidades prácticas para la reformulación de programas de salud con el enfoque de DSS.

5- Contenidos, métodos y tareas de aprendizaje.

Como parte del marco referencial de educación de adultos elegido, se contemplaron 4 elementos metodológicos básicos a desarrollar, éstos son: la elección de contenidos, la exploración de las expectativas de los actores, la vinculación de los contenidos con la afectividad y emociones de los participantes y la utilización de actividades prácticas en el desa- rrollo del proceso educativo.

a) Contenidos: Los contenidos principales fueron:

1.- Aspectos introductorios del enfoque de determinantes sociales .Se utilizó como base de trabajo el informe final de la comisión de determinantes sociales de la de la OMS.

2- Inequidades en salud y trabajo. Se utilizó como base de trabajo el informe de la CDSS referente a empleo (Emconet) ${ }^{11}$.

3-Metodología de incorporación de la perspectiva de DSS en los programas de salud. Basado en la GEE de los programas de salud, la cual fue desarrollada por la subsecretaría de salud pública.

b) Exploración de expectativas: A los participantes se les solicitó llenar una cartulina con su respectiva expectativa acerca del curso, ésta fue leída en voz alta y categorizada en papel kraft. El resultado fue rediscutido con el plenario, quienes validaron la interpretación de las expectativas.

c) Afectividad y emociones: Se buscó generar un vínculo afectivo con la temática a partir de identificación catártica, utilizando como vehículo la visualización de la película "La Cuadrilla", de Ken Loach, la cual trata de un grupo de operarios de vías de ferrocarril en la época de la privatización de este servicio en Gran Bretaña en la década de los 90.

d) Praxis: Se trabajó en grupos de discusión y talleres en torno a las distintas temáticas. Luego de la visualización de la película contestaron de manera individual una pauta de trabajo, que exploró las consecuencias de la precariedad laboral, la vulnerabilidad de los trabajadores y el rol de la distribución desigual del poder entre empleador y trabajador, luego de lo cual se dialogó en un grupo de no mas de 8 personas y extrajeron sus propias conclusiones, las cuales presentaron en plenario para la reflexión general. En lo que se refiere al trabajo de la GEE, se les solicitó elegir un programa de salud pertinente a su trabajo, para luego identificar grupos vulnerables, visualizar barreras y facilitadores del acceso al programa, analizar críticamente la 
teoría del programa, vincular los determinantes sociales con las problemáticas pesquisadas y, finalmente, elaborar objetivos y prioridades para el rediseño del programa

\section{7.- Evaluación y Cierre}

Cuantitativamente la evaluación se realizó al finalizar el primer día y se repitió al final del curso. Se les pidió calificar en una escala de 4 ítems (desde muy bueno a malo); la metodología docente, el interés en el tema y los contenidos. Para ello debieron emitir un juicio personal en papel kraft, especialmente diseñado. Se realizará, además, una evaluación cualitativa (oral y escrita), en la cual se iniciará una reflexión en torno de la pregunta ¿Qué aprendimos? ¿Qué les gustó?, ¿Qué no les gustó o qué mejoraría?

\section{8.- Sistematización de la Experiencia}

Si bien no se eligió ex -ante un marco de investigación cualitativa con el cual registrar y explorar los hallazgos de la experiencia educativa, la metodología de recopilación de información consideró el registro manual literal de las opiniones y percepciones vertidas por los participantes durante la experiencia (verbal y/o escrita), la que luego fue analizada y codificada según el método de codificación abierta según lo descrito para una primera etapa en la teoría fundada $^{12}$. Desde la perspectiva de la validez del estudio, la triangulación fue llevada a cabo por los tres investigadores y tuvo lugar en el momento de la categorización de la información y en la elaboración de las conclusiones.

Tabla 1. Resumen de expectativas y categorías.

\begin{tabular}{|l|l|}
\hline Categoría & Ejemplos de frases textuales \\
\hline $\begin{array}{l}\text { Adquirir conocimientos y conceptos } \\
\text { relevantes de los DSS }\end{array}$ & $\begin{array}{l}\text { "Conceptualizar los determinantes sociales de la salud." } \\
\text { "Obtener mayor conocimiento de los determinante sociales de la } \\
\text { salud." }\end{array}$ \\
\hline $\begin{array}{l}\text { Conocer con el propósito de evaluar la } \\
\text { aplicabilidad del enfoque de DSS }\end{array}$ & $\begin{array}{l}\text { "Comprender en la totalidad lo que significan los determinantes socia- } \\
\text { les, como nos afectan, pero también si mis conocimientos, mi forma de } \\
\text { proceder ante alguna determinada situación puede paliar o disminuir } \\
\text { algún determinante." } \\
\text { "Conocer los objetivos generales del curso y ver si son aplicables a la } \\
\text { realidad." }\end{array}$ \\
\hline $\begin{array}{l}\text { Conocer para aplicar el enfoque de } \\
\text { DSS en la práctica diaria }\end{array}$ & $\begin{array}{l}\text { "Conocer la forma en que se puede aplicar el enfoque de determinan- } \\
\text { "Comprender la teoría y aplicar la metodología de determinantes } \\
\text { sociales." }\end{array}$ \\
\hline Dinamismo & "Que todos podamos participar", "que sea dinámico." \\
\hline No expresa expectativas & "No tengo expectativas." \\
\hline
\end{tabular}

\section{RESULTADOS}

Los resultados serán analizados asumiendo a ambos grupos como miembros de un mismo colectivo, pero se mencionarán algunos elementos distintivos de cada grupo cuando sea pertinente.
1- Etapa II y III: Apertura/Introducción y Vínculo

\section{A- Expectativas}

Las frases textuales fueron codificadas y categorizadas, y se resumen en la Tabla 1. 
Tabla 2. Resumen de identificación de DSS estructurales e intermediarios de trabajadores del film, categorizados según modelo OMS.

\begin{tabular}{|c|c|c|c|}
\hline Pregunta & Ámbito & Frases y/o palabras textuales & Categorías OMS \\
\hline \multirow{10}{*}{$\begin{array}{l}\text { ¿Qué determinantes } \\
\text { de la Salud de los } \\
\text { trabajadores de la } \\
\text { cuadrilla (estructu- } \\
\text { rales e intermedia- } \\
\text { rios) usted observa? }\end{array}$} & \multirow{6}{*}{ Estructurales } & $\begin{array}{l}\text { "Situación político económica", "modelo } \\
\text { económico", "doctrina política", "contexto } \\
\text { socioeconómico" }\end{array}$ & $\begin{array}{l}\text { Contexto político-eco- } \\
\text { nómico }\end{array}$ \\
\hline & & "Contexto cultural", "cohesión social" & Contexto cultural \\
\hline & & "Política social" & Política social \\
\hline & & $\begin{array}{l}\text { "Mercado laboral", "legislación laboral", } \\
\text { "inestabilidad laboral", "vulnerabilidad de } \\
\text { los trabajadores", "precariedad laboral". }\end{array}$ & Mercado laboral \\
\hline & & $\begin{array}{l}\text { "Poder", "abuso de poder", "clase social", } \\
\text { "jerarquía social". }\end{array}$ & Jerarquía social \\
\hline & & $\begin{array}{l}\text { "Posición socioeconómica", "posición } \\
\text { social", "nivel socioeconómico bajo", "ocu- } \\
\text { pación/ingreso", "inequidad en ingresos", } \\
\text { "nivel educacional", "nivel de ingreso". }\end{array}$ & $\begin{array}{l}\text { Posición socioeconó- } \\
\text { mica }\end{array}$ \\
\hline & \multirow{4}{*}{ Intermediario } & $\begin{array}{l}\text { "Condiciones materiales (vivienda)", "haci- } \\
\text { namiento". }\end{array}$ & Condiciones materiales \\
\hline & & $\begin{array}{l}\text { "Factores psicosociales", "miedo", "presión } \\
\text { laboral y familiar", "tipos de familia", "esta- } \\
\text { do civil", "stress laboral y familiar", "tipos } \\
\text { de convivencia". } \\
\text { "Condiciones laborales (desprotección)", } \\
\text { "desprotección social", "modalidad de } \\
\text { contrato". }\end{array}$ & Factores psicosociales \\
\hline & & $\begin{array}{l}\text { "Condiciones inseguras de trabajo", "malas } \\
\text { condiciones laborales sin áreas para descan- } \\
\text { so", "problemas de higiene laboral". }\end{array}$ & $\begin{array}{l}\text { Factores físico-bioló- } \\
\text { gicos }\end{array}$ \\
\hline & & $\begin{array}{l}\text { "Alimentación inadecuada, conductas/ali- } \\
\text { mentación". }\end{array}$ & $\begin{array}{l}\text { Conductas asociadas a } \\
\text { salud }\end{array}$ \\
\hline
\end{tabular}

\section{$B$ - Taller cine y trabajo}

Posterior a la visualización de la película se les solicitó completar la pauta individual de trabajo, para luego reunirse en grupo para dialogar las respuestas y construir conclusiones grupales. Las preguntas y los resultados gru- pales se resumen en el cuadro 2 y 3 , en ellos se agrega una columna, elaborada por los autores, donde se clasifica las expresiones vertidas por los participantes, según el modelo de determinantes de la OMS y de Emconet, respectivamente. 
Tabla 3. Resumen de identificación de características y mecanismos causales relacionados al deterioro de la salud de los trabajadores del film, categorizados según modelo Emconet.

\begin{tabular}{|c|c|c|c|}
\hline Pregunta & Frase textual & Categoría conceptual & Categoría Emconet \\
\hline \multirow{3}{*}{$\begin{array}{l}\text { ¿Qué carac- } \\
\text { terísticas del } \\
\text { empleo y/o del } \\
\text { trabajo pudiesen } \\
\text { estar afectando } \\
\text { la salud de estos } \\
\text { trabajadores? }\end{array}$} & $\begin{array}{l}\text { "Inestabilidad laboral, no exis- } \\
\text { tían derechos laborales." }\end{array}$ & Inseguridad/ inestabilidad & Condiciones de empleo \\
\hline & $\begin{array}{l}\text { "Inseguridad laboral (subcon- } \\
\text { tratación sin capacidad de to- } \\
\text { mar decisiones, ni negociar)", } \\
\text { "política empresarial dictato- } \\
\text { rial", "vulnerabilidad" }\end{array}$ & $\begin{array}{l}\text { Desequilibrio de poder } \\
\text { Empleador/ Trabajador }\end{array}$ & Condiciones de empleo \\
\hline & $\begin{array}{l}\text { "Falta de protección social y } \\
\text { desintegración social." }\end{array}$ & $\begin{array}{l}\text { Mayor vulnerabilidad } \\
\text { social }\end{array}$ & Condiciones de empleo \\
\hline \multirow{4}{*}{$\begin{array}{l}\text { ¿Qué mecanis- } \\
\text { mos causales } \\
\text { pudiesen estar } \\
\text { involucrados en } \\
\text { el deterioro de su } \\
\text { salud? }\end{array}$} & $\begin{array}{l}\text { "Condiciones inseguras } \\
\text { (accidentes, enfermedades y } \\
\text { muerte)." }\end{array}$ & $\begin{array}{l}\text { Mayor exposición a riesgos } \\
\text { físicos y psicológicos }\end{array}$ & Condiciones de trabajo \\
\hline & $\begin{array}{l}\text { "Trabajo bajo presión, ausen- } \\
\text { cia de beneficios y protección } \\
\text { laboral, alimentación inadecua- } \\
\text { da sin valoración de derechos } \\
\text { sindicales." "Inseguridad e } \\
\text { inestabilidad laboral llevan a } \\
\text { la enfermedad mental, stress y } \\
\text { aceptación de mayor riesgo." }\end{array}$ & $\begin{array}{l}\text { Aumento de conductas de } \\
\text { riesgo }\end{array}$ & $\begin{array}{l}\text { Comportamientos asocia- } \\
\text { dos a la salud }\end{array}$ \\
\hline & $\begin{array}{l}\text { "La precariedad laboral influye } \\
\text { en las relaciones de amistad y } \\
\text { solidaridad", "crisis social y } \\
\text { personal." }\end{array}$ & $\begin{array}{l}\text { Deterioro de las relaciones } \\
\text { sociales (confianza, cohe- } \\
\text { sión social) }\end{array}$ & Factores psicosociales \\
\hline & $\begin{array}{l}\text { "Recurso humano insufi- } \\
\text { ciente", "sobre exigencia", } \\
\text { "pérdida de vida familiar", "es- } \\
\text { trés", "fatiga", "carga horaria } \\
\text { afectividad familiar y tipo de } \\
\text { jornada". }\end{array}$ & $\begin{array}{l}\text { Sobrecarga física y mental } \\
\text { Deterioro de relaciones } \\
\text { familiares }\end{array}$ & Factores psicosociales \\
\hline
\end{tabular}

\section{2.- Etapa III: Aplicación práctica}

\section{A- Resultados del desarrollo de la GEE}

En todos los grupos que eligieron el programa de salud ocupacional, la vulnerabilidad fue asociada a la desprotección en que se encuentran los trabajadores que no acceden a los beneficios de la ley 16.744, que permite a los trabajadores recibir prestaciones médicas, en especies y dinero frente a accidentes laborales y enfermedades profesionales (Tabla 4). La inequidad también se relaciona e identifica con aquellos grupos que, por encontrase en una situación de pobreza y aislamiento geográfico, 
Tabla 4. Identificación y caracterización de personas en situación de inequidad.

\begin{tabular}{|l|l|l|l|}
\hline Programa de Salud & $\begin{array}{l}\text { Grupo en situación de } \\
\text { inequidad }\end{array}$ & Características del grupo & $\begin{array}{l}\text { Situación de } \\
\text { inequidad }\end{array}$ \\
\hline & $\begin{array}{l}\text { Trabajadores informales } \\
\text { aplicadores de pesticidas }\end{array}$ & $\begin{array}{l}\text { "Trabajo temporal, sin contrato, en } \\
\text { su mayoría hombres". }\end{array}$ & $\begin{array}{l}\text { "No cubiertos por } \\
\text { la ley 16.744" }\end{array}$ \\
\cline { 2 - 4 } & $\begin{array}{l}\text { Trabajadoras de faenas } \\
\text { agrícolas temporeras } \\
\text { Informales }\end{array}$ & $\begin{array}{l}\text { "Mujeres en su mayoría, sin contra- } \\
\text { to, provenientes del sector rural, con } \\
\text { una alta tasa de analfabetismo." }\end{array}$ & $\begin{array}{l}\text { "No cubiertos por } \\
\text { la ley 16.744" }\end{array}$ \\
\cline { 2 - 5 } Programa de Salud & $\begin{array}{l}\text { Trabajadores afiliados al } \\
\text { ISL en calidad de obreros } \\
\text { de la provincia de Ranco }\end{array}$ & $\begin{array}{l}\text { "Desinformación de derechos", } \\
\text { "Dificultades de acceso a centros } \\
\text { prestadores de servicios de salud por } \\
\text { aislamiento geográfico". }\end{array}$ & $\begin{array}{l}\text { "Inequidad en el } \\
\text { acceso a las pres- } \\
\text { taciones de la ley } \\
16.744 "\end{array}$ \\
\cline { 2 - 5 } & $\begin{array}{l}\text { Trabajadores informales del } \\
\text { sector agropecuario provin- } \\
\text { cia de Ranco }\end{array}$ & $\begin{array}{l}\text { "En su mayoría hombres, provenien- } \\
\text { tes del sector rural, bajos ingresos, } \\
\text { sin contrato, con una tasa alta de } \\
\text { analfabetismo y entre los } 20 \text { y } 50 \\
\text { años de edad". }\end{array}$ & $\begin{array}{l}\text { "No cubiertos por } \\
\text { la ley 16.744" }\end{array}$ \\
\cline { 2 - 5 } & $\begin{array}{l}\text { Trabajadoras informales de } \\
\text { casa particular }\end{array}$ & $\begin{array}{l}\text { "Mujeres jefas de hogar, sin contra- } \\
\text { to". }\end{array}$ & $\begin{array}{l}\text { "No cubiertos por } \\
\text { la ley 16.744" }\end{array}$ \\
\hline
\end{tabular}

no pueden acceder a las prestaciones de este seguro. Entre las barreras más importante se mencionan; pobreza, nivel educacional, fallas del fiscalizador, falta de competencias de los trabajadores (particularmente en intersectorialidad), dificultades en contar con información que les permita cuantificar o caracterizar a los grupos vulnerables, prácticas discriminatorias de las mutuales administradoras del seguro, aislamiento geográfico y los déficit de conectividad, además del modelo económico imperante. (Figura 2)

En cuanto al desarrollo de la GEE, llama la atención que se centraron inmediatamente en la definición de grupos vulnerables, antes de analizar críticamente las características y evidencia científica del programa de salud. En estos grupos aparecieron dificultades importantes para conceptualizar las etapas de un programa y en la comprensión de lo que llamamos "evidencia científica". Por otro lado, los pasos finales de la GEE fueron desarrolladas sólo parcialmente, identificando al Servicio Nacional de la Mujer, la Dirección del Trabajo y la Seremi de Educación, como los sectores prioritarios con los cuales coordinar acciones. El paso final que buscaba definir objetivos y prioridades para el rediseño del programa no alcanzó a ser desarrollado por los participantes.

\section{3- Etapa IV de Evaluación final}

\section{A- Evaluación cualitativa}

Frente a las preguntas: ¿Qué aprendimos?, ¿Qué les gustó? y ¿Qué no les gustó o que mejoraría?, la Tabla 5 resume los hallazgos. 


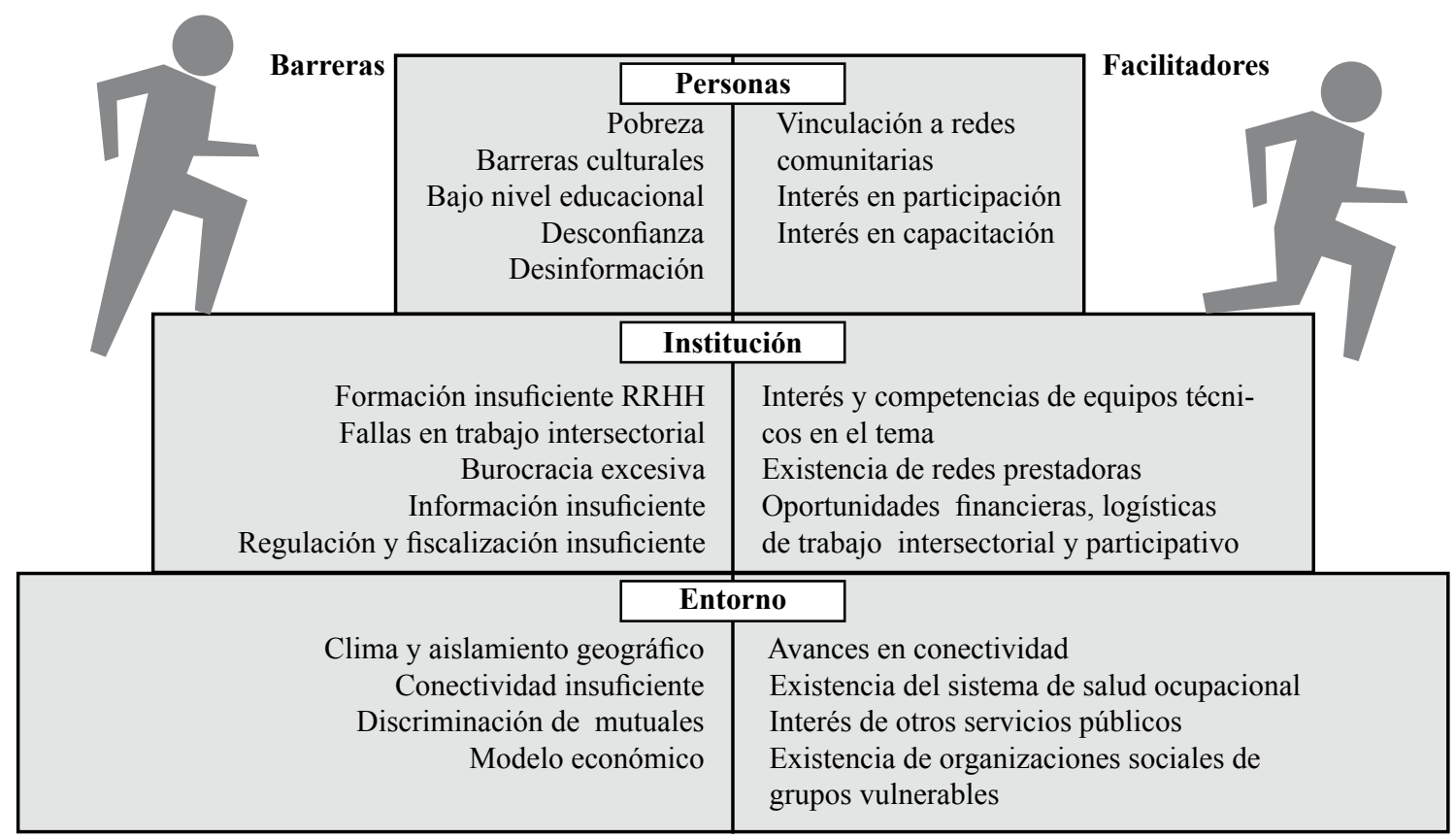

Figura 2. Resumen de barreras y facilitadores, según tipo.

Tabla 5. Resumen evaluación cualitativa.

\begin{tabular}{|c|c|c|}
\hline Ámbito & Positivo & Negativo \\
\hline $\begin{array}{l}\text { Comprensión y aplicación } \\
\text { de enfoque }\end{array}$ & $\begin{array}{l}\text { Categoría: La mejor comprensión del rol del } \\
\text { sistema de salud y del sentido del trabajo. } \\
\text { Frases textuales: } \\
\text { "valorar las pequeñas cosas que hacemos todos } \\
\text { los días", "hoy estamos entendiendo mejor lo } \\
\text { que estamos programando." } \\
\text { "lo más triste de todo es llegar a la conclu- } \\
\text { sión de que los que menos tienen son los más } \\
\text { vulnerables." }\end{array}$ & $\begin{array}{l}\text { Categoría: Falta de sentido } \\
\text { del enfoque de DSS en el } \\
\text { trabajo diario. } \\
\text { Frases textuales: } \\
\text { "No se puede hacer mucho } \\
\text { pues todo viene normado" }\end{array}$ \\
\hline $\begin{array}{l}\text { Valoración de la metodo- } \\
\text { logía }\end{array}$ & $\begin{array}{l}\text { Categoría: Valoración del diálogo y participa- } \\
\text { ción. } \\
\text { Frases textuales: } \\
\text { "La dinámica permite conocerse y trabajar } \\
\text { juntos", "hay espacio para desarrollarse mucho } \\
\text { más.” } \\
\text { Mayor participación “...descentralizada, viene } \\
\text { de nosotros, de abajo." }\end{array}$ & $\begin{array}{l}\text { Categoría: Ansiedad y proble- } \\
\text { mas con la metodología. } \\
\text { Frases textuales: } \\
\text { "Sería bueno que explicaran } \\
\text { la metodología al inicio" "no } \\
\text { estamos acostumbrados" } \\
\text { "se necesitan más presenta- } \\
\text { ciones" }\end{array}$ \\
\hline
\end{tabular}




\section{B-Evaluación cuantitativa}

El último día de trabajo, el 100\% de los participantes evaluó la metodología y los contenidos del curso en la categoría de bueno o muy bueno, con un proporción similar para cada categoría, evidenciándose un cambio respecto a la evaluación del primer día, en el cual las categorías regular y malo alcanzaron un $35 \%$ (30\% y $5 \%$, respectivamente), la categoría bueno, un $40 \%$ y la muy bueno, un $25 \%$. En cuanto al interés en el curso se observa similar cambio (ver Figura 3).

\section{DISCUSION}

Los resultados expuestos muestran el desarrollo, por parte de los participantes, de la capacidad de identificar determinantes sociales estructurales e intermediarios, en torno a una determinada problemática. Lograron definir grupos vulnerables de distintos programas de salud y elaborar un marco de barreras y facilitadores que resultan atingentes a las problemáticas estudiadas, lo cual indica la adopción de algunos de los conceptos tratados. Respecto al desarrollo de análisis crítico y de habilidades para rediseñar un determinado programa, los logros son menos claros, sólo el primer grupo pudo identificar espacios de cambio en su trabajo cotidiano. En parte, la falta de tiempo no permitió desarrollar la parte final de la GEE que apuntaba a desarrollar estos ámbitos de aprendizajes.

Las evaluaciones del curso nos muestran la valoración de la participación, del espacio de encuentro generado y del aporte a la comprensión de los contenidos, no sin generar tensiones y resistencias a la misma. Por otro lado, cuando observamos la capacidad de identificar grupos vulnerables, determinantes de salud y posibles mecanismos causales, observamos una gran coincidencia con los marcos de análisis de los informes de la CDSS y Emconet. Temas como la precariedad laboral, vulnerabilidad, distribución desigual del poder, los efectos sobre la salud mental y familiar, la mayor exposición a riesgos y su vinculación con las características del empleo y trabajo, no fueron presentados sino después del análisis de los grupos, lo que nos indica la capacidad del cine no sólo de vincular afectivamente a los participantes, sino también de permitir la comprensión en profundidad de la temática tratada, en concordancia con algunos enfoques de la teoría del film ${ }^{13}$.

Por otro lado, la gran mayoría de los participantes de ambos grupos, había escuchado acerca del tema de los DSS, pero la participación en procesos de capacitación en torno al tema fue más bien excepcional. Esto nos habla del esfuerzo declarativo del sector salud en impulsar el tema de los determinantes sociales, pero también respecto a las dificultades en la "traducción" conceptual y práctica del enfoque. Cuando distintas publicaciones analizan y miran retrospectivamente procesos de alfabetización en distintos lugares del planeta, uno de los aprendizajes más universales en re-

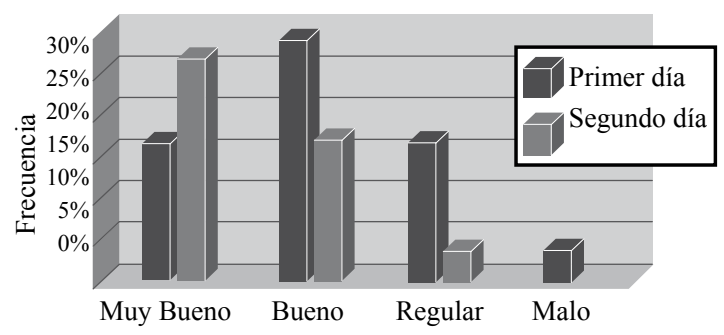

Figura 3. Evaluación interés en el curso. Fuente: Elaboración propia

lación con el desarrollo de la lecto-escritura, es la relevancia de comprender primero el significado que ésta tiene y tendrá en el mundo en que las personas transcurren ${ }^{14}$. En el caso de la implementación de un enfoque transformador como el de determinantes sociales de la salud, sin pasar por los significados, experiencias $\mathrm{y}$ afectos de quienes deben implementarlos, puede producir resistencia y escepticismo, la que en parte es reconocida en esta experiencia, 
que dificulta el proceso de cambio. Recoger estos pareceres desde una perspectiva dialógica implica estar dispuesto a analizar críticamente y en conjunto, la pertinencia de los conceptos involucrados y construir el acuerdo de lo que esto significará para cada grupo humano y territorio. El desarrollo institucional de esta competencia dialógica, parece ser uno de los desafíos pendientes en el proceso de implementación de este enfoque de DSS.

Tal espacio de diálogo genera y generará brechas de competencias de las organizaciones $\mathrm{y}$ de las personas que las constituyen. ¿Cómo se trabaja con otros sectores que miran las problemáticas sociales desde otras perspectiva?, ¿Qué habilidades del ámbito de la comunicación debemos desarrollar?, ¿Cómo se trabaja con el mundo laboral, con una cuota baja de poder de transformación desde el sector?, son interrogantes que surgieron de diversas formas durante el transcurso de este espacio educativo. Por otro lado, no es raro encontrar la solicitud a los trabajadores sanitarios de fomentar la participación. Cuando este trabajo participativo no es comprendido como un proceso, en un entorno complejo y con abiertas desigualdades en la distribución del poder y de los recursos de diversos tipos, tampoco se comprenden las brechas que emergen ante tal solicitud.

Finalmente el proceso educativo implementado permitió identificar en profundidad aspectos determinantes de inequidad sanitaria en diversos niveles de la realidad local de la Seremi de Salud de la Región de Los Ríos. Entre ellas podemos mencionar informalidad y precariedad del trabajo, aislamiento geográfico, inequidad de ingresos y de educación, entre las más importantes. Otro ámbito de inequidad identificado es la distinción entre obreros y empleados, por una parte, y la falta de regulación y fiscalización, por otra, de los ya protegidos por el seguro laboral. Si bien se valora positivamente la presencia del seguro y los prestadores asociados a éste, no es menos cierto que la discrecionalidad de las mutuales administradoras del seguro al elegir sus asegurados (o elegir a las empresas), se identifica como factor de inequidad, lo cual mantiene abierto un ámbito de estudio y análisis respecto de las fallas de nuestro sistema de salud ocupacional. Por otra parte, el sistema de salud y sus trabajadores aparece como uno de los facilitadores más importantes, aún cuando la falta de trabajo intersectorial y las fallas en las competencias de los trabajadores, para abordar las problemáticas, son brechas aún por resolver.

\section{REFERENCIAS}

1. CDSS. Subsanar las desigualdades en una generación. Resumen analítico informe final. Ginebra: OMS; 2008.

2. PNUD. Informe sobre Desarrollo Humano 2009. Superando barreras: Movilidad y desarrollo humano. Nueva York: PNUD; 2009.

3. MINSAL. Informe I: Estrategia para la integración de los determinantes sociales y equidad en los programas de salud del ministerio de salud de Chile. Santiago de Chile: OPS/Minsal; 2008.

4. CARLOS MATUS. Plan y Estrategia. Editorial Universitaria S.A., Santiago de Chile 1972.

5. TORRES, R. De la alfabetización al aprendizaje a lo largo de toda la vida: Tendencias, temas y desafíos de la educación de personas jóvenes y adultas en América Latina y el Caribe. Síntesis del Reporte Regional. Hamburgo: Unesco/Institute for Lifelong Learning; 2009.

6. WALTER, P. Philosophies of Adult Environmental Education. Adult Education Quarterly 2009; 60 (1): 3-25.

7. BHOLA, H. Aspectos curriculares de la postalfabetización y la educación continua de los neoalfabetas. Hamburgo :IUE-Unesco; 1984.

8. SCHMELKES, S. Necesidades básicas de aprendizaje de los adultos en América Latina en UnescoUnicef, La educación de adultos en América Latina ante el próximo siglo. Santiago: Unesco-Unicef; 1994.

9. VELLA, J.Taking learning to Task: Creative Strategies for Teaching Adults. The Jossy-Bass Higher an Adult Education. Jossey-Bass Publishers: San Francisco; 2000.

10. MINSAL. Guía para analizar equidad en el acceso y los resultados de los programas y su relación con los determinantes sociales de la salud. Santiago de Chile; 2009.

11. BENACH J, MUNTANER, $\mathrm{C}$ and SANTANA, V. Employment Conditions and Health Inequalities Employment Conditions Knowledge Network (Emconet). Final Report. Ginebra: OMS/CDSS/EM- 
CONET; 2007

12. STRAUSS, A. Y CORVIN, J. Basic of qualitative research: Grounded theory procedures and thechniques. London: Sage Publications; 1990.

13. ORTIGOSA, S. La Educación en valores a través del vine y las artes. Revista Iberoamericana de Educación 2002; (29): 157-175.

14. SCHMELKES, S. y KALMAN J. La educación de adultos: estado del arte. Hacia una estrategia alfabetizadora para México. San Angel: INEA; 1996.

Usted puede comentar éste y otros artículos publicados en la Revista Chilena de Salud Pública, enviando un correo electrónico a revistasp@med.uchile.cl 